\title{
Multivariate data handling in the study of rat behavior: An integrated approach
}

\author{
Maurizio Casarrubea, Filippina Sorbera, and Giuseppe Crescimanno \\ Università di Palermo, Palermo, Italy
}

\begin{abstract}
The aim of the present article is to provide a methodological description of various approaches to multivariate data handling in the study of rodent behavior. To this purpose, 42 male Wistar rats were tested in an open field, and their behavior was recorded through a digital video camera for a subsequent analysis by means of a software coder. After a preliminary evaluation of descriptive features such as durations and percent distributions, we carried out different kinds of multivariate approaches represented by stochastic, cluster, adjusted residual, and T-pattern analyses. In the attempt to depict behavior in a straightforward way, the results of each analysis were graphically illustrated through path diagrams, dendrograms, histograms, and tree-shaped T-patterns. Path diagrams showed a clear behavioral convergence toward immobile sniffing; dendrograms highlighted three different dyadic clusters: walking/climbing, immobile-sniffing/immobility, and paw-licking/grooming; adjusted residuals confirmed, for the same patterns, highly significant association values; finally, T-pattern analysis showed a highly recurring temporal sequence of events encompassing walking, climbing, immobile sniffing, and immobility. Such results, drawing attention to specific behavioral patternings, strengthen and extend previous findings on rodent behavior. We suggest that T-pattern analysis, integrated with other multivariate approaches, can provide a more detailed and complete rat behavior representation, very different from classical quantitative approaches.
\end{abstract}

Multivariate analysis (MVA) represents a useful tool for investigating human or animal behavior. As was suggested in a landmark article by Spruijt and Gispen (1984), it is possible to explore behavior from different points of view only through evaluation of interrelations among behavioral elements. Indeed, MVA encompasses techniques aimed at examination of data sets with more than one variable - that is, the analysis of interrelations among studied items. Medium to high requirements in terms of computational capabilities, together with a lack of suitable computer programs, have delayed application of multivariate data handling for a long time. During the last 2 decades, because of the increasing development and diffusion of personal computers and specifically developed software, this problem has been gradually overcome. In recent articles, through MVA, we have emphasized new aspects of rat behavior in various experimental models, such as the open field and hole board (Casarrubea, 2008; Casarrubea, Sorbera, \& Crescimanno, 2006, 2008, 2009a, 2009b). Also, MVA has been utilized to illustrate different features of rat responses in the elevated plus-maze (Cruz, Frei, \& Graeff, 1994; Espejo, 1997), in the hot plate (Espejo \& Mir, 1993, 1994), and in the forced swimming test (Lino-de-Oliveira, De Lima, \& Carobrez, 2005). By means of such methods, animal grooming activities (Kalueff \& Tuohimaa, 2004), aggressiveness-related responses (Aguilar et al., 2002; Aguilar et al., 2003; Mos,
Olivier, \& van der Poel, 1987), and social (Van Den Berg, Van Ree, \& Spruijt, 1999) and genetic (Takahashi, Kato, Makino, Shiroishi, \& Koide, 2006) influences on behavior have been described as well. In an interesting article, Hemerik, Bukovinszky, Gols, van Lenteren, and Vet (2006) proposed a thoughtful depiction of a specific multivariate approach in the study of parasitoid foraging behavior. In brief, the authors used a maximum likelihood method to detect sudden changes (i.e., breakpoints) in their event log files. Moreover, they used different statistical tests within and between matrices, followed by a T-pattern analysis, to demonstrate animals' activity modifications. In a recent article, we showed that rat exploratory behavior was influenced by external acoustic stimulation (Casarrubea et al., 2008) and, therefore, that modifications in event log files could be induced by specific independent variables. On the other hand, unexpected changes in behavioral streams could also depend on subjects' internal causes. Thus, use of a breakpoint should be carefully considered, since it could depend on the experimental design and animals used. We suggest that the structural analysis of behavior does not necessarily need identification of breakpoints. Rather, T-pattern analysis, integrated with different multivariate approaches, can provide a more detailed and complete rat behavior representation.

As has been diffusely illustrated before, different multivariate approaches have been employed to analyze a be-

M. Casarrubea, m.casarrubea@unipa.it 
Table 1

Ethogram of Rat Exploratory Behavior

\begin{tabular}{ll}
\hline Walking & Rat moves around sniffing the environment. \\
Climbing & Rat maintains an erect posture leaning against the Plexiglas wall. \\
Rearing & Rat maintains an erect posture without leaning against the wall. \\
Paw licking & Rat licks or grooms its paws. \\
Grooming & Rat rubs its face or licks the body, combing the fur by fast movements of incisors. \\
Immobile sniffing & Rat sniffs the environment, firmly standing on the ground. \\
Immobility & A fixed posture is maintained. \\
\hline
\end{tabular}

havior. However, an integrated method, able to detect and represent the behavioral structure from diversified multivariate points of view, has not been previously proposed. Thus, the aim of the present study was to (1) provide a step-by-step methodological description of four different multivariate approaches (stochastic, cluster, adjusted residuals, and T-pattern analyses) to study rat behavior, (2) show the feasibility of their synergical integration in depicting rodent behavior under different points of view, and (3) present results in a easily understandable fashion, by means of specific graphical representations associated with each multivariate approach. To these purposes, Wistar rats were tested in a classical open-field apparatus, and their behavior was recorded by means of a digital video camera and analyzed through a personal computer equipped with suitable software. The open-field apparatus was chosen because it offered a simple and widely accepted approach for studying different features of rodent behavior, such as locomotor and exploratory activities, as well as the possible effects of different drugs.

\section{METHOD}

\section{Apparatus and Subjects}

The open-field apparatus used in this experiment consisted of a $40 \times 40 \mathrm{~cm}$ square Plexiglas arena. Forty-two male SPF Wistar rats (Morini, Italy), 50-55 days old, weighing 220-250 g, were tested. The animals were housed individually in a room maintained at $23^{\circ} \pm 1^{\circ} \mathrm{C}$, with lights on at 7:00 a.m. and off at 7:00 p.m. Water and food (standard diet, Morini, Italy) were available ad lib.

\section{Procedure}

All experimental observations were carried out in a single day from 8:00 a.m. to 1:00 p.m. The rats were transported to the testing room within their home cages to minimize possible transfer effects. The animals were allowed to acclimate for $30 \mathrm{~min}$ far from the openfield apparatus to avoid possible visual and/or olfactory influences. Also, the temperature was maintained equal to that of the housing room $\left(23^{\circ} \pm 1^{\circ} \mathrm{C}\right)$. Each rat was placed in the open field and was allowed to freely explore the environment for $150 \mathrm{sec}$. After each test, the apparatus was carefully cleaned with ethylic alcohol (70\%) to remove possible scent cues left from the preceding animal. The experiments were recorded through a digital video camera (Creative Optia AF) and stored in ".avi" format in a personal computer for subsequent analyses.

The experiment was carried out in accordance with the European Communities Council Directive 86/609/EEC concerning the protection of animals used for experimental scientific purposes.

\section{Terminology}

Terms such as item (Takahashi et al., 2006), element (Spruijt \& Gispen, 1984), or even just behavior (van Lier, Coenen, \& Drinkenburg, 2003) are commonly used to indicate discrete behavioral units encompassed in a given behavioral sequence. However, since the word pattern (Casarrubea et al., 2006, 2008, 2009a; Espejo \& Mir, 1993 ; Lino-de-Oliveira et al., 2005) is common in ethological language to designate such events, in the present article, pattern or behavioral pattern will indicate a given unit within the ethogram (see below).

\section{Data Analysis}

The first step was the construction of an ethogram - that is, a formal list containing descriptions of behavioral patterns. After that, video files were observed by means of a software coder (The Observer; Noldus Information Technology, Wageningen, The Netherlands). The ethogram reported (Table 1) is a simplified version of the one presented in our previous article (Casarrubea et al., 2008).

Following the preliminary steps above, various descriptive approaches were carried out. In the present study, to differentiate frequent patterns from infrequent ones, percent distributions were evaluated and represented through a pie chart. Moreover, the mean duration of each behavioral pattern's first appearance was calculated as well.

To evaluate relationships among patterns, different multivariate analyses were carried out and integrated: stochastic analysis, cluster analysis, adjusted residual analysis, and T-pattern analysis. A detailed step-by-step procedure of such approaches will be presented in the following sections.

Multivariate data handling. Behavioral elements were coded from the collected video files. Through the relevant option available within the Observer, transitions from an element to another one were traced in a transition matrix (TM). Figure 1 exemplifies a TM with three behavioral patterns.

\section{Example of Transition Matrix}

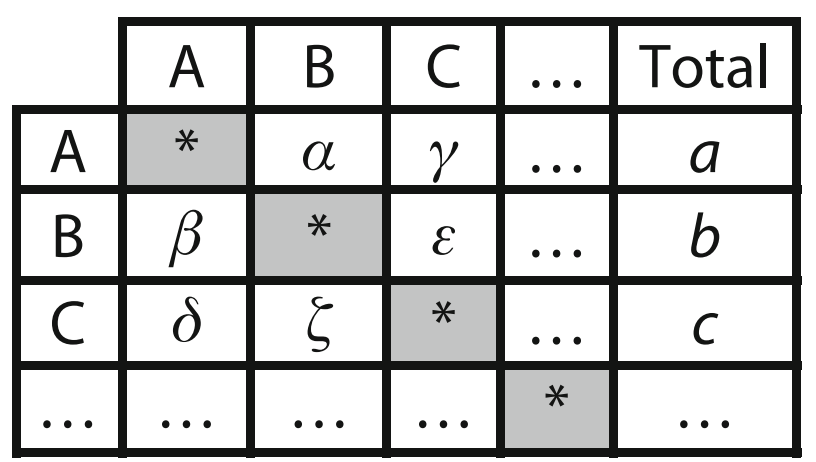

Figure 1. Example of a transition matrix. A, name of a given behavioral pattern " $A$ "; $B$, name of a given behavioral pattern "B"; $C$, name of a given behavioral pattern " $C$ "; $\alpha$, number of transitions from Pattern $A$ to $B ; \gamma$, number of transitions from Pattern A to $\mathrm{C} ; \beta$, number of transitions from Pattern $\mathrm{B}$ to $\mathrm{A} ; \varepsilon$, number of transitions from Pattern B to $\mathrm{C} ; \delta$, number of transitions from Pattern $\mathrm{C}$ to $\mathrm{A}$; $\boldsymbol{\zeta}$, number of transitions from Pattern $\mathrm{C}$ to $\mathrm{B}$; $\boldsymbol{a}$, total occurrences of Behavioral Pattern $\mathrm{A}$; $\boldsymbol{b}$, total occurrences of Behavioral Pattern B; $c$, total occurrences of Behavioral Pattern C. *Structural zeros (i.e., an antecedent element cannot be followed by itself). 
Within a TM, the following critical conditions should always be observed: (1) a minimum number of transitions at least five times the number of selected behavioral patterns (Spruijt \& Gispen, 1984); (2) overall number of empty cells no more than $20 \%$ (Casarrubea et al., 2008, 2009a); and (3) number of variables (i.e., matrix rows and columns names) at least three times less the number of subjects used (Espejo \& Mir, 1993; Short \& Horn, 1984). The main purpose of the requirements above is to ensure stable correlations among described patterns. Indeed, as was noticed by Spruijt and Gispen, poorly filled matrices represent a serious issue in a multivariate analysis.

In the present article, TMs for each subject were calculated and summed together, obtaining a total TM. It represented the starting point for following multivariate analyses, except T-pattern analysis, which must be carried out by means of a specific software on event $\log$ files. Individual TMs, along with the resulting total TM, were fully processed by means of a software for matrix manipulation and analysis (Matman, Version 1.1; Noldus Information Technology).

TM elaboration. Basically, a TM (Figure 1) is a table filled with hundreds of numbers representing shifts among behavioral elements according to a selected ethogram. In most cases, a TM is quite useless in its form, since it is often difficult, even for experienced researchers, to appreciate each transition meaning. As a consequence, a TM should be expressed in a more intuitive way.

Stochastic analysis. The main purpose of the stochastic approach proposed is to emphasize probabilistic relationships and provide a direction of transitions among behavioral patterns. Different methods are available to characterize direction of transitions from a pattern to the others. For instance, considering Patterns A and $\mathrm{B}$ of the exemplifying TM in Figure 1, an interesting method uses the $(\alpha-\beta) /(\alpha+\beta)$ formula (Mos et al., 1987). However, by means of our stochastic approach, both directions and probabilities among patterns can be expressed together. Hence, on the basis of the relative frequencies of transitions among patterns, a TM can be transformed into a probability matrix.

Four qualifications need to be strictly respected within a probability matrix: (1) Each row must sum to 1, (2) all patterns must be between 0 and 1, (3) 0 means no transition between two given patterns in the originating TM, and (4) switching probability from a pattern to all others is 1 . A probability matrix is a normal from-to matrix that can be graphically expressed through a path diagram, where different transition probabilities are represented by connecting arrows of different thicknesses.

Cluster analysis. The aim of cluster analyses is to represent similarities among patterns. A total TM needs to be transformed into a similarity matrix through a specific algorithm allowing the identification of behavioral clusters.

Considering the exemplifying TM in Figure 1, the similarity between Patterns A and B is assessed by the following formula (Mos et al., 1987):

$$
S=(\alpha / a+\beta / b+\beta / a+\alpha / b) 50,
$$

where $S$ represents the similarity value between two given patterns, calculated on the basis of the overall number of reciprocal transitions, and 50 is a normalization factor.

It is very important to stress that, unlike TMs and probability matrices, a similarity matrix is not a from-to matrix, and each cell is not at all representative of a behavioral transition, but it is a correlation $(S)$ between two given patterns. For this reason, a similarity matrix is a half-matrix from which a dendrogram can be obtained. A dendrogram is a graphical tree representation showing how similar some patterns, encompassed in a given ethogram, are to one another.

Adjusted residuals analysis. To compare TMs, Tavaré and Altham (1983) proposed a correction factor - the so-called gamma correction - to multiply by the chi-square value. On the other hand, an elegant method for assessing the significance of cells within matrices was used by Everitt (1977) and Spruijt (Spruijt \& Gispen, 1984) and, after them, by different authors (Casarrubea et al., 2008, 2009a; Van Den Berg et al., 1999; Vanderschuren, Spruijt, Hol, Niesink, \& Van Ree, 1996; van Lier et al., 2003). Through this method, a TM is transformed into a matrix containing adjusted residuals. In summary, for each given transition, an adjusted residual represents the difference between the observed cell value and an expected one. Positive residuals indicate transitions occurring more often than expected; negative residuals represent transitions occurring less often than expected. Adjusted residuals were computed through Matman Version 1.1 (Noldus Information Technology) - that is, a specific software for matrix manipulation and analysis. A consistent advantage of adjusted residuals is that they can be expressed according to a $Z$ distribution so that $p$ values can be easily found in a common $Z$ table: Values $\geq+1.96$ and $\leq-1.96$ reveal significant transitions $(p \leq .05)$. Thus, positive residuals with values $\geq+1.96$ show transitions occurring significantly more often than expected, and negative residuals, with values $\leq-1.96$, indicate transitions occurring significantly less often than expected. Like probability matrices, adjusted residuals matrices can be illustrated by means of path diagrams (Spruijt, 1992; Spruijt \& Gispen, 1984; Van Den Berg et al., 1999; Vanderschuren et al., 1996). However, our approach illustrates adjusted residuals through histograms, using positive and negative bars (Casarrubea et al., 2008, 2009a).

T-pattern analysis. The aim of a T-pattern analysis is to evaluate significant relationships (i.e., critical intervals) among events in the course of time (Magnusson, 2000). A T-pattern analysis was carried out through the specific Theme software (Patternvision and Noldus Information Technology). This program is able to perform, on selected data sets obtained from the software coder, a recursive test, checking the distribution of every combination of events along a specific time window (Magnusson, 2000). Figure 2 depicts a hypothetical T-pattern of three elements. If the time lag of an event sequence is not randomly distributed, a simple T-pattern is detected. In subsequent steps, such a first-level T-pattern is processed again, and if there is a significant temporal relation with other events, a higher order T-pattern is obtained, and so on, following a bottom-up process (Magnusson, 2000). T-patterns represent complex structures emerging from an analysis, where any time unit may be used. A detailed description of the theory, concepts, and procedures behind T-pattern analysis can be found in different articles by Magnusson $(2000,2004)$.

By means of an appropriate option available within the Theme software, it is possible to join event log files into a single data set. In our experiment, a T-pattern detection procedure was carried out on 42 joined event log files. Since our video files had a temporal resolution of $1 \mathrm{sec}=25$ frames, each video $(150 \mathrm{sec})$ was 3,750 frames long and, as a consequence, 42 joined event log files resulted in a whole data set of $157,500(3,750 \times 42)$ frames.

西

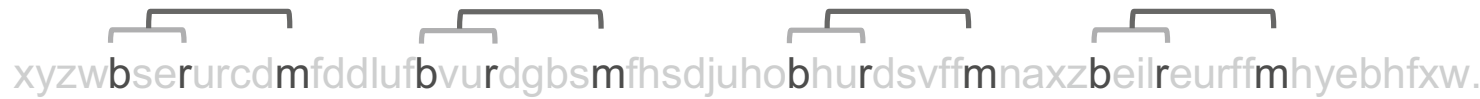

xyzwbserurcdmfddlufbvurdgbsmfhsdjuhobhurdsvffmnaxzbeilreurffmhyebhfxw...

Figure 2. Exemplifying T-pattern encompassing three hypothetical events. Bold arrow, observational period $\left(T_{0}-T_{x}\right)$ consisting of 70 events (black letters). The T-pattern represented above $(b, r, m)$ becomes evident if all the other behavioral occurrences are left out (gray letters) from the whole observational time line. See the text for details concerning the tree T-pattern representation. 
The search parameters, after such a joining procedure, were (1) minimum occurrences (minimum number of times a pattern had to occur to be detected) $=5$, (2) significance level (maximum accepted probability that any critical interval relationship would occur by chance $=.005$, and (3) lumping factor (forward/backward probability that an event or pattern would exceed the given value to be detected) $=.90$.

Several recent studies have utilized T-patterns to analyze behavioral modifications in neuropsychiatric conditions (Kemp et al., 2008; Lyon \& Kemp, 2004), route-tracing stereotypy in the mouse (Bonasera, Schenk, Luxenberg, \& Tecott, 2008), feeding behavior in broilers (Martaresche, Le Fur, Magnusson, Faure, \& Picard, 2000; Merlet et al., 2005), and interactions among human subjects and animal subjects or among humans and robots (Kerepesi et al., 2005; Kerepesi, Kubinyi, Jonsson, Magnusson, \& Miklósi, 2006). Currently, no studies have focused on the application of T-pattern analysis in rat behavioral structure. In the present research, in addition to the stochastic, cluster, and adjusted residual analyses, a T-pattern analysis was carried out in the attempt to (1) discover possible T-patterns in rats exploring a novel environment, (2) show the feasibility of the integration of T-pattern analysis with other multivariate approaches, and (3) demonstrate the effectiveness of T-pattern analysis application in behavioral observations on rats.

\section{RESULTS}

Our findings emphasize, by joining different approaches, how to describe a behavior not only in quantitative terms, but also, importantly, in its qualitative aspects. After assessments of durations and percent distributions, the results of stochastic, cluster, adjusted residual, and T-pattern analyses will be presented.

\section{Descriptive Analyses}

Percent distributions of patterns, represented through a pie chart (Figure 3), demonstrated that the behavioral pattern with the highest percent value was walking, followed by immobile sniffing and climbing. Moreover, these three patterns encompassed roughly $80 \%$ of the whole behav-

\section{Percent Distribution}

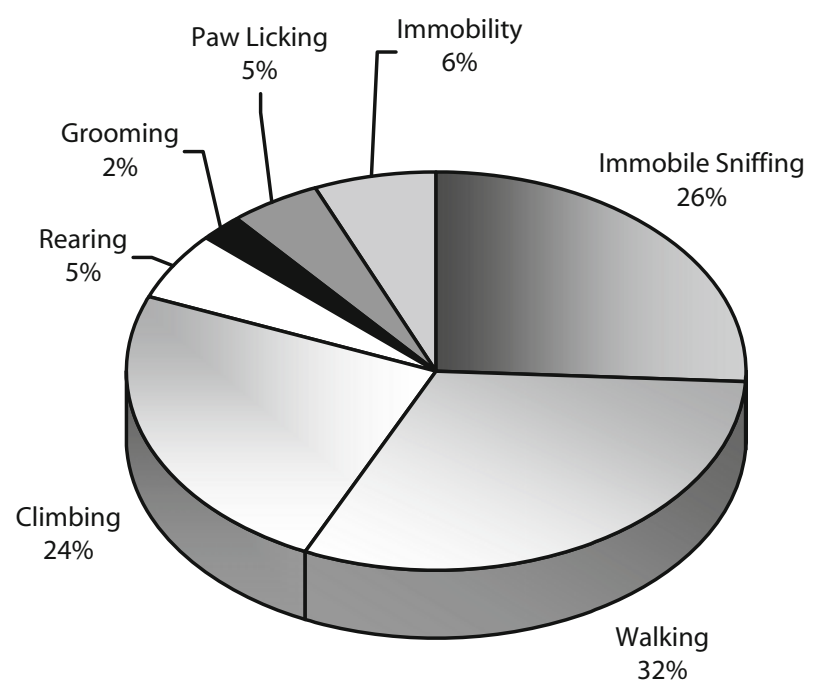

Figure 3. Percent distribution of each behavioral element.
Table 2

Mean Duration (in Seconds; With Standard Errors) of Each Behavioral Pattern's First Appearance

\begin{tabular}{lcc}
\hline \multicolumn{1}{c}{ Pattern } & $M$ & $S E$ \\
\hline Climbing & 1.306 & 0.145 \\
Rearing & 1.800 & 0.352 \\
Grooming & 2.490 & 0.533 \\
Walking & 3.557 & 0.573 \\
Immobile sniffing & 6.136 & 0.781 \\
Paw licking & 6.420 & 1.324 \\
Immobility & 17.725 & 2.526 \\
\hline
\end{tabular}

Note-The data were obtained from the analysis of 42 animals.

ioral structure. All the remaining patterns showed percent values below $10 \%$. Mean duration $\pm S E$ of each pattern's first appearance showed values below 1 sec for rearing and climbing, from 2 to $4 \mathrm{sec}$ for walking and grooming, about $6 \mathrm{sec}$ for paw licking and immobile sniffing, and more than $10 \mathrm{sec}$ for immobility (Table 2).

\section{Stochastic Analysis}

The path diagrams in Figure 4 show all the transitions among patterns from a probabilistic perspective. Fortytwo total transitions have been illustrated: 22 showed a probability range going from 0 to $.09,9$ from .1 to .24 , 8 from .25 to .49 , and 3 from .50 to 1 . To avoid complicated representations, transition probabilities $\leq .09$ (Figure 4A) and $\geq .10$ (Figure 4B) were represented separately.

\section{Cluster Analysis}

The dendrogram in Figure 5 depicts similarity values among patterns on the basis of reciprocal number of transitions. Through $S$ values, obtained from the half-similarity matrix, three dyadic clusters have been detected: $S=110.9$ (walking/climbing), $S=102.2$ (immobility/immobilesniffing), and $S=66.1$ (paw-licking/grooming). Rearing maintained an outside position because of its $S=49.1$.

\section{Adjusted Residual Analysis}

The histograms in Figure 6 represent adjusted residuals - that is, transitions occurring significantly more often or less often than expected. According to the $Z$ table, three different $p$ levels were selected: $p \leq .05(*), p \leq .001$ $(* *)$, and $p \leq .0001(* * *)$. Positive $Z$ values pointing at $p \leq .0001$ were detected between immobile sniffing and immobility, immobility and immobile sniffing, walking and climbing, and paw licking and grooming. No transitions occurred significantly less often than expected ( $p \leq$ $.0001)$. Significant positive and negative associations with $p \leq .05$ and $p \leq .001$ were observed among several other behavioral patterns.

\section{T-Pattern Analysis}

A total of 24 independent T-patterns were detected. In detail, 14 were dyadic, 9 encompassed three events, and 1 T-pattern encompassed four events (Figure 7). The tree representation in Figure 8A illustrates the latter, highly recurring T-pattern characterized by the string ((walking climbing)(immobile-sniffing immobility)), which occurred 23 times (Figure 8B and 8C) during the whole 
A Path Diagrams
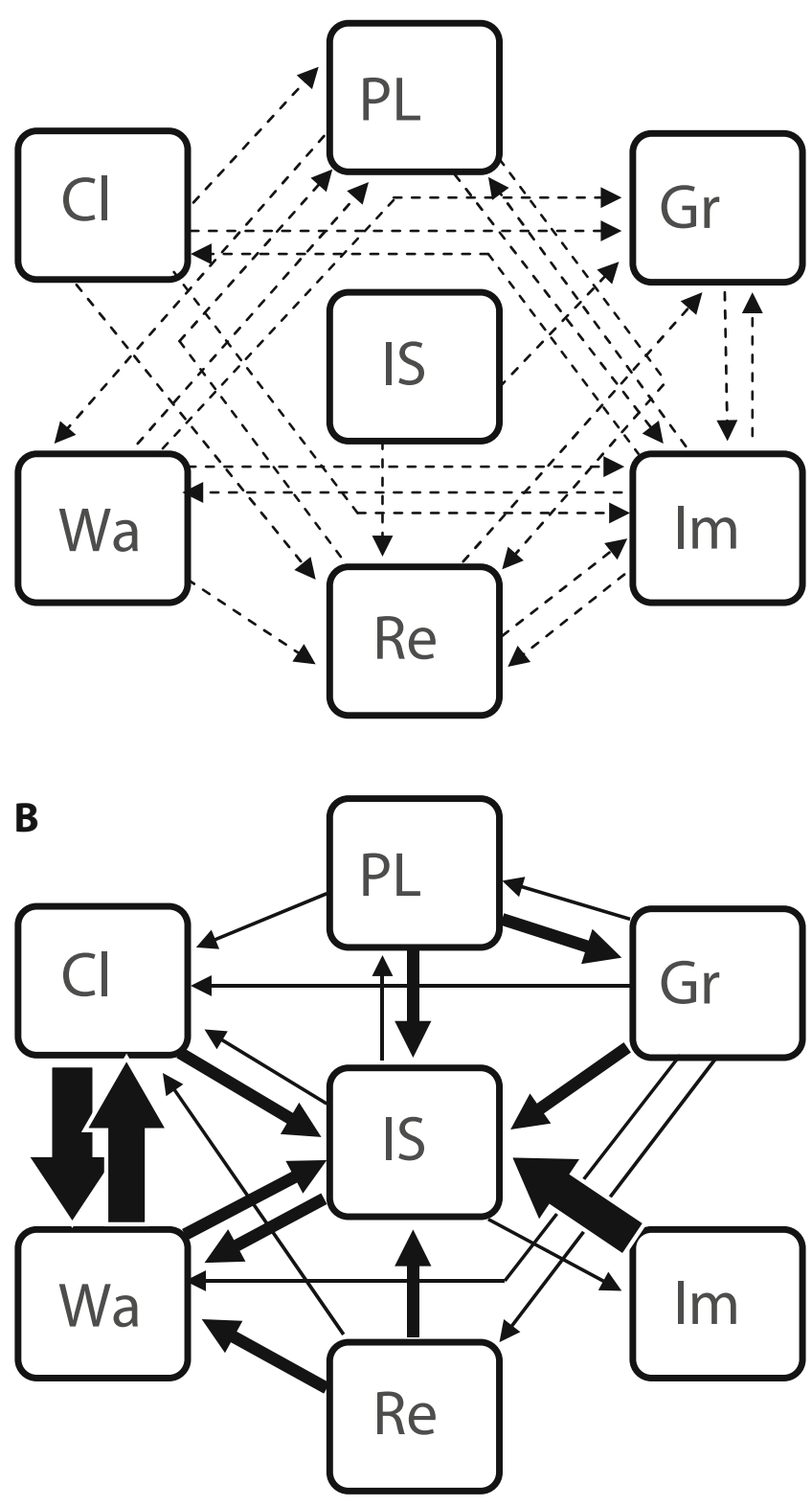

Probability Ranges
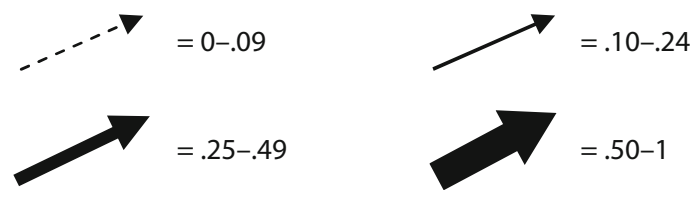

Figure 4. Path diagram representing transitional probabilities among behavioral elements. At the bottom, the selected probability ranges used to draw probability vectors of different thicknesses are shown $(\mathrm{A}=0-.09 ; \mathrm{B}=.10-.24, .25-.49, .50-1)$. The data were obtained from the analysis of 42 animals. PL, paw licking; $\mathrm{Cl}$, climbing; Gr, grooming; IS, immobile sniffing; Wa, walking; Im, immobility; Re, rearing. 


\section{Dendrogram}

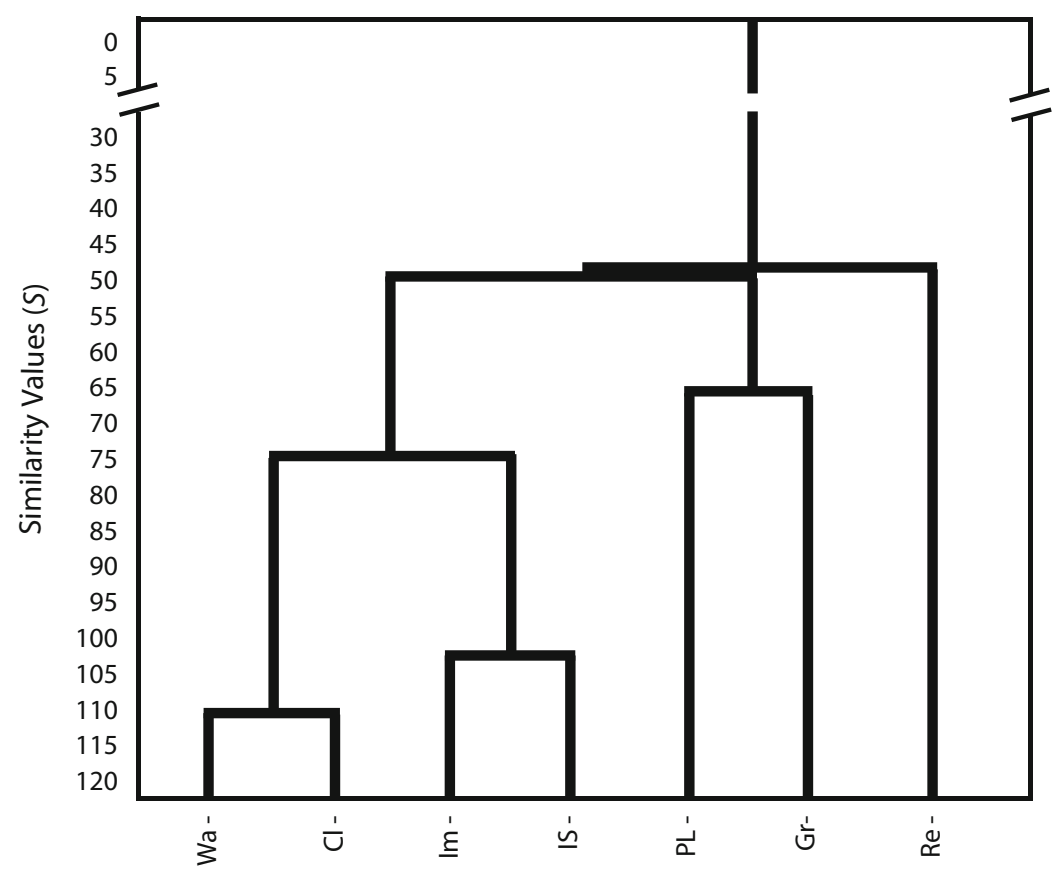

Figure 5. Dendrogram representing similarities among behavioral elements. Data were obtained from the analysis of 42 animals. Wa, walking; $\mathbf{C l}$, climbing; Im, immobility; IS, immobile sniffing; PL, paw licking; Gr, grooming; Re, rearing.

\section{Adjusted Residuals}
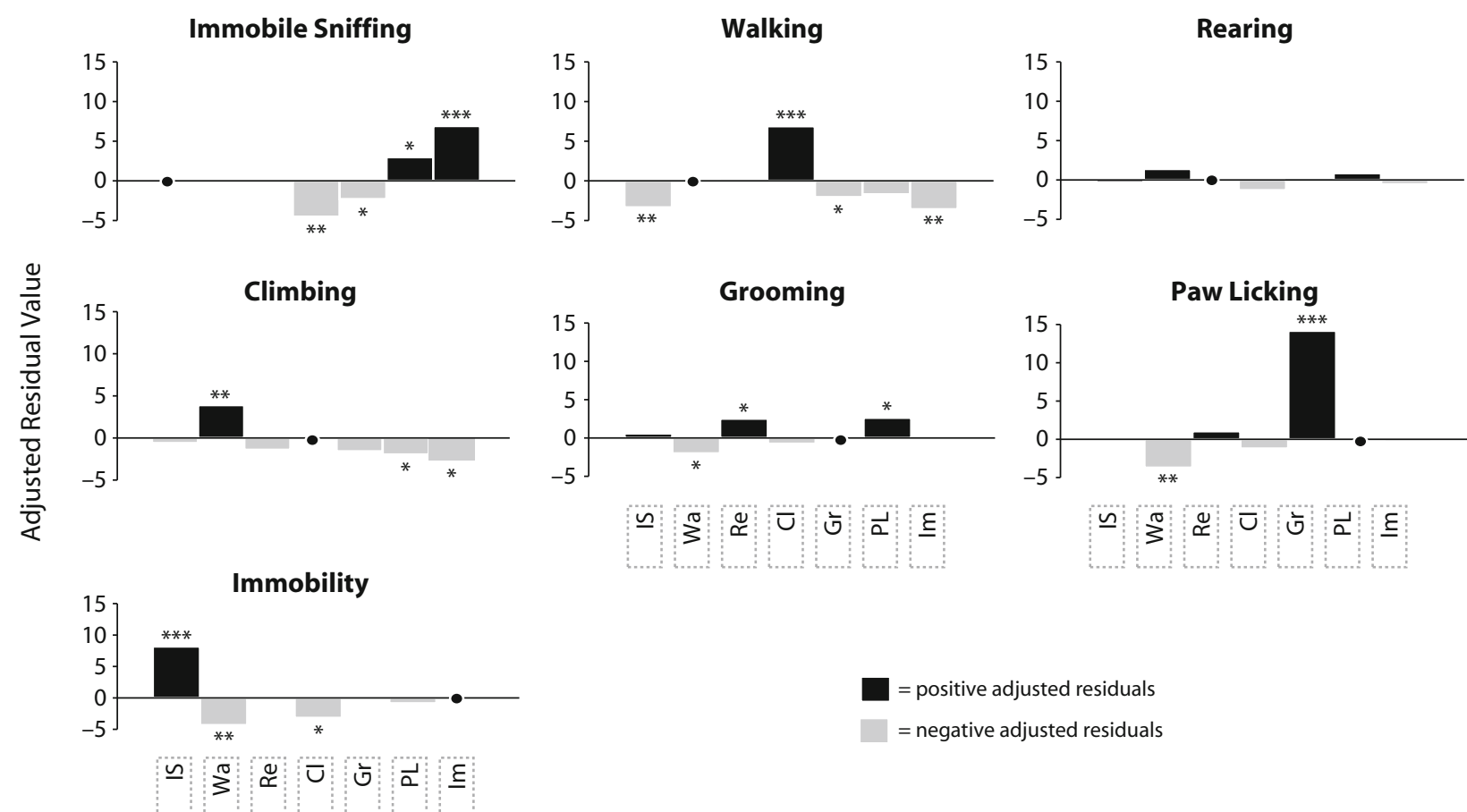

= positive adjusted residuals

$=$ negative adjusted residuals

Figure 6. Adjusted residuals representing the differences between the observed values and the expected ones. Heading of each panel: behavioral element antecedent to the ones indicated along the $x$-axis; $y$-axis, adjusted residuals values. Black bars, positive residuals (i.e., transitions occurring more often than expected); gray bars, negative residuals (i.e., transitions occurring less often than expected). According to the $Z$ table, three different $p$ levels have been selected: ${ }^{*} p \leq .05 ;{ }^{* *} p \leq .001 ;{ }^{* * *} p \leq .0001$. Black dots along $x$-axes, structural zeros (i.e., an antecedent element cannot be followed by itself). Data were obtained from the analysis of 42 animals. IS, immobile sniffing; Wa, walking; Re, rearing; Cl, climbing; Gr, grooming; PL, paw licking; Im, immobility. 
Pattern Length Distribution-Average for 10 Random Runs

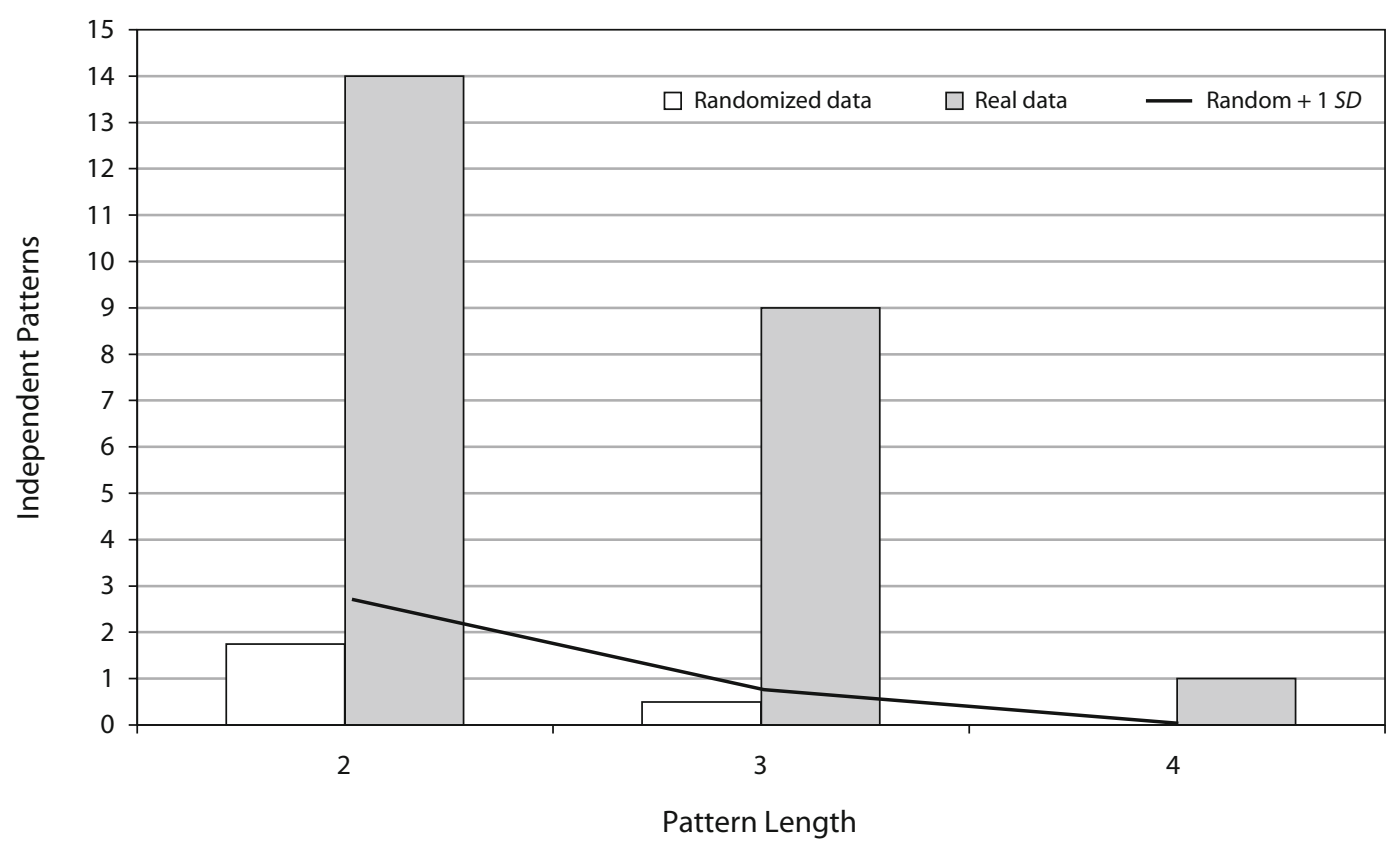

Figure 7. Comparison between randomized and real data on an average of 10 random runs. The $x$-axis gives the pattern length; $y$-axis, number of different independent T-patterns. Black line shows the mean number of patterns in the randomized data plus one standard deviation.

T-Pattern

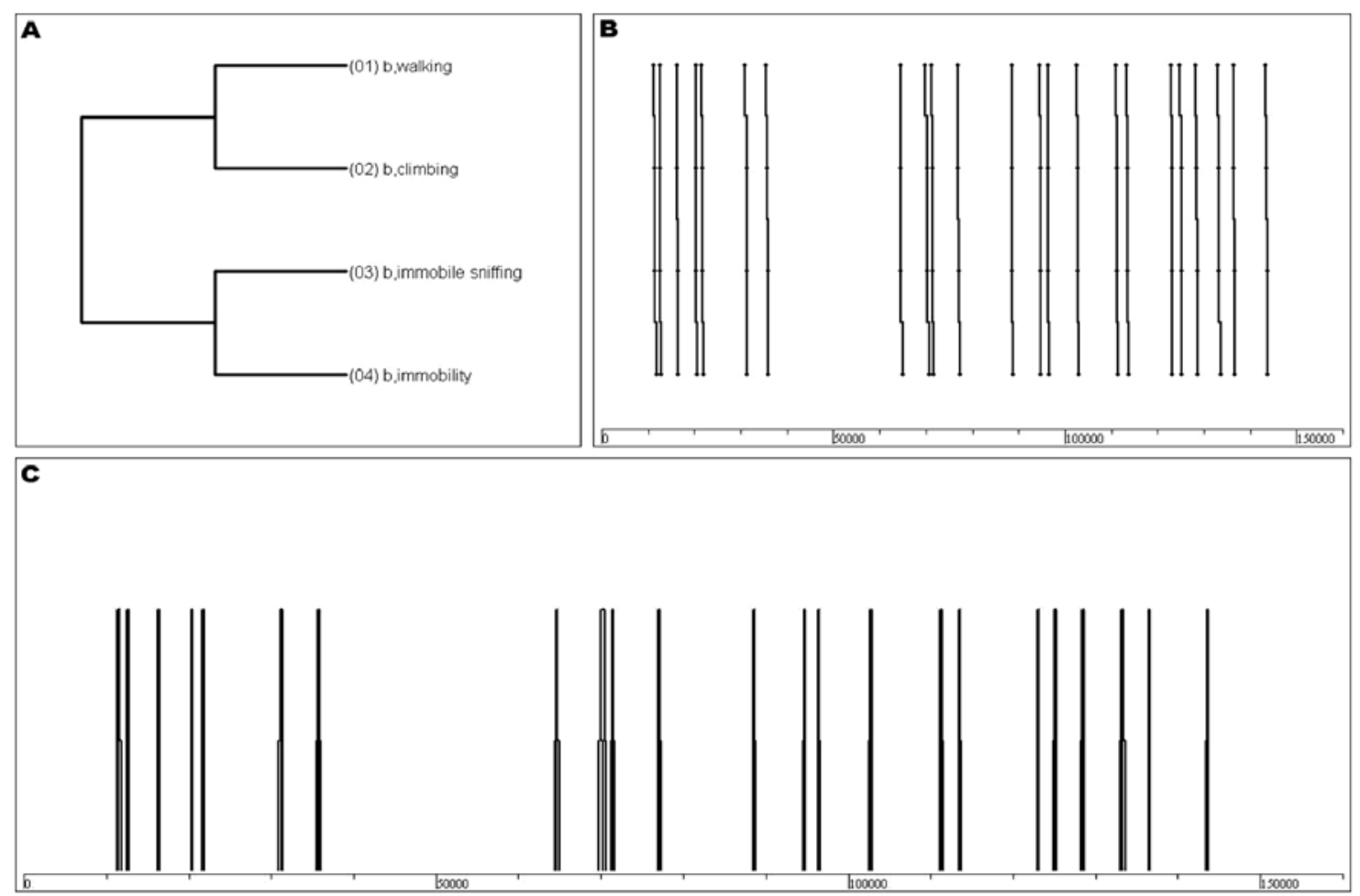

Figure 8. T-patterns represent the existence of significant relationships among events during the whole observational period. (A) T-pattern tree graph, encompassing walking (01), climbing (02), immobility (03), and immobile sniffing (04). (B) All occurrences of T-pattern events, shown through a connection graph. (C) Twenty-three occurrences of this significant $(p \leq .005)$ T-pattern, shown on time baseline $(157,500$ frames). Data were obtained from the analysis of 42 animals. 
observational period. Notably, with reference to search parameters, if different values (such as 3 or 10) within minimum occurrences field were used and/or if lumping value was unchecked, similar findings could be obtained. To statistically assess our data, we used the same search parameters to detect possible T-patterns in randomized data, obtaining significant results for 5,10 , or 100 randomization runs. Figure 7 compares randomized and real data.

\section{DISCUSSION}

In this article on methodology, a step-by-step description of four different multivariate approaches, aimed at the study of rat behavior in an open field, has been provided. Stochastic, cluster, adjusted residual, and T-pattern analyses have been integrated and respectively associated with graphical depictions, so as to provide detailed and synergical representations of behavior. Also, "classical" analyses of patterns' percent distributions and durations have been carried out. However, besides results provided by descriptive evaluations, differences in terms of usefulness and applicability between these approaches and MVAs have to be underlined. Durations (Table 2) and percent distributions (Figure 3), as well as frequencies and latencies, present detailed quantifications of a behavior. However, a drawback is that each pattern, although carefully described and quantified, represents a disjointed element. This is a pivotal aspect differentiating standard quantitative evaluations of behavior from MVAs. For instance, a pie chart (Figure 3) clearly shows that immobility and immobile sniffing represent, together, one third of the whole behavior. Moreover, durations (Table 2) show high values for both immobility and immobile sniffing. However, relationships among these patterns with the whole behavioral architecture cannot be evidenced by a descriptive approach alone. An important aspect of the MVA is the ability to analyze interrelations among all behavioral units. The study of these interrelations should be considered an essential component of a behavioral investigation, because they allow a thoughtful depiction of qualitative dynamics. Still, with respect to immobile sniffing, in different recent articles, we have demonstrated, by means of MVA, that behavioral flow is directed toward this behavioral element. Thus, immobile sniffing is suggested to play a crucial role in behavior organization (Casarrubea et al., 2008, 2009a) and, from a purely qualitative perspective, as "the behavioral expression of an ongoing selection process that . . . could be ethologically defined an awaiting selection pattern" (Casarrubea et al., 2008). The present findings extend our knowledge of immobile sniffing, since we demonstrated that only medium to high transition probabilities are directed toward immobile sniffing, as illustrated in path diagrams (Figures 4A and 4B).

Graphical expressions of probability matrices are stochastic path diagrams. They provide an important advantage consisting of simultaneous presentation of both direction and transition probability. Path diagrams, showing behavioral flow from a probabilistic point of view, make possible a straightforward understanding even at a first glance. In Figure 4, all behavioral transitions are reported. Nonetheless, a separate representation of probabilities (Figures 4A and 4B) has been presented in order to avoid an intricate illustration.

Dendrograms are representations of a data-clustering algorithm. In our analysis, an aggregative hierarchical procedure has been followed. However, independently of the clustering algorithm used, dendrograms are useful representations, since they allow the partitioning of whole behavior into segments (i.e., clusters). In such a way, a better and immediate understanding of relationships between different groups of patterns is available. Figure 5 focuses on three different groups of behaviors. Considering $S$ values, representing similarity on the basis of the reciprocal number of transitions between patterns, the walking/climbing cluster showed important relations with immobility/immobile-sniffing but not with paw-licking/ grooming. However, it is essential to note that such tree representations do not express a direction of transition at all; as a consequence, dendrograms and path diagrams should be considered closely linked. Moreover, even though dendrograms and path diagrams represent intuitive graphical representations, these approaches should be completed with a further statistical support: the adjusted residuals (Casarrubea et al., 2008, 2009a).

Adjusted residual histograms are graphical representations of matrices containing adjusted residuals. Albeit adjusted residual matrices have been expressed by means of path diagrams in different articles (Spruijt, 1992; Spruijt \& Gispen, 1984; Van Den Berg et al., 1999; Vanderschuren et al., 1996), we propose that graphical expression through histograms should be preferred for two reasons: First, confounding representations with stochastic path diagrams may be generated; second, for an inexperienced reader, graphical representation of negative residuals (i.e., transitions occurring significantly less often than expected) could give rise to misunderstandings from a conceptual point of view. On the contrary, histograms representation (Figure 6) plainly shows adjusted residuals, providing a clear-cut difference between positive and negative values. A further interesting aspect is that adjusted residuals provide statistical information "within matrices," which, in turn, can be compared to obtain additional statistical validations (Casarrubea et al., 2008; Vanderschuren et al., 1996).

The benefit of path diagrams, dendrograms, and adjusted residual histograms is that they represent patternings among behavioral elements in an intuitive graphical fashion. However, these representations originate from the same matrix elaborations. Matrices are very sensitive to noise; that is, uncommon behavioral elements should be removed before running the analysis. Indeed, as was reported in the Data Analysis section, inadequately filled matrices represent a critical issue. Moreover, it should be taken into account that stochastic, cluster, and adjusted residual analyses, together with all kinds of analyses based on matrices, represent observational 
time windows, like "snapshots" of an entire period. It is important to underline that a behavioral event, albeit uncommon, could be crucial for the whole behavioral structure and, as a consequence, its exclusion is always arbitrary. T-pattern detection represents events during session time, and importantly, it does not require an a priori noise reduction.

Both T-pattern graphical illustration and cluster analysis results are visualized through tree representations. However, cluster analyses are based on similarities among events, whereas the tree structure provided by Theme emphasizes the existence of significant relationships in the course of time. T-pattern analysis (Figure 8) confirms and further extends other MVA results (Figures 4,5 , and 6) concerning relationships between walking and climbing, immobile sniffing and immobility, and paw licking and grooming. Indeed, walking, climbing, immobile sniffing, and immobility remain highly related to one another along time (Figures $8 \mathrm{~B}$ and $8 \mathrm{C}$ ). On the other hand, paw licking and grooming are detached from this highly recurrent T-pattern. For this reason, paw licking and grooming might represent different behavioral categories, not only for their phenomenological characteristics, but also, and importantly, for timing control central mechanisms.

\section{Conclusion}

Percent distributions or durations are useful from a descriptive point of view, since they provide precise information concerning each investigated item. However, descriptive approaches are quite ineffective in depicting behavior's most important feature- that is, relationships among observed behavioral elements. The possibility to characterize each behavioral pattern through even thousands of numbers does not imply the possibility to use those numbers to figure out what the behavior is in its wholeness. As a consequence, descriptive approaches to behavioral studies should be partnered with MVAs. Even a swift comparison among path diagrams, dendrograms, adjusted residuals, and T-patterns emphasizes how different multivariate approaches strengthen one another in representing animal behavior: Path diagrams and dendrograms show patternings among behavioral elements from probabilistic and aggregative points of view, respectively; adjusted residuals provide a strong statistical support; and T-patterns represent the behavioral structure in the course of time. The present research demonstrates that different approaches to multivariate data handling can be used to depict animal behavior from different synergical points of view, greatly beyond what the human eye can intuitively interpret. Further studies are in progress in our laboratory to improve the knowledge of rat behavioral structure in different experimental paradigms.

\section{AUTHOR NOTE}

This work was supported by a grant from Università degli Studi di Palermo, Palermo, Italy. The authors are grateful to Magnus S. Magnusson, University of Iceland, Reykjavik, for his help concerning the use of the Theme software and its applicative purposes. Correspondence concerning this article should be addressed to M. Casarrubea, Depart- ment of Experimental Medicine, Human Physiology Section, University of Palermo, Corso Tukory No. 129, 90134 Palermo, Italy (e-mail: m.casarrubea@unipa.it).

\section{REFERENCES}

Aguilar, R., Gil, L., Flint, J., Gray, J. A., Dawson, G. R., Driscoll, P., ET AL. (2002). Learned fear, emotional reactivity and fear of heights: A factor analytic map from a large $\mathrm{F}_{2}$ intercross of Roman rat strains. Brain Research Bulletin, 57, 17-26.

Aguilar, R., Gil, L., Gray, J. A., Driscoll, P., Flint, J., Dawson, G. R., ET AL. (2003). Fearfulness and sex in F2 Roman rats: Males display more fear though both sexes share the same fearfulness traits. Physiology \& Behavior, 78, 723-732.

Bonasera, S. J., Schenk, A. K., Luxenberg, E. J., \& Tecott, L. H. (2008). A novel method for automatic quantification of psychostimulant-evoked route-tracing stereotypy: Application to Mus musculus. Psychopharmacology, 196, 591-602.

Casarrubea, M. (2008). Multivariate analyses for the study of behavior: An integrated approach. In A. J. Spink, M. R. Ballintijn, N. D. Bogers, F. Gricco, I. W. S. Loijens, L. P. J. J. Noldus, \& G. Smit (Eds.), Measuring Behavior 2008: 6th International Conference on Methods and Techniques in Behavioral Research (pp. 231-233). Wageningen, The Netherlands: Noldus Information Technology.

Casarrubea, M., Sorbera, F., \& Crescimanno, G. (2006). Effects of 7-OH-DPAT and U 99194 on the behavioral response to hot plate test, in rats. Physiology \& Behavior, 89, 552-562.

Casarrubea, M., Sorbera, F., \& Crescimanno, G. (2008). Multivariate analysis of the modifications induced by an environmental acoustic cue on rat exploratory behavior. Physiology \& Behavior, 93, 687-696.

Casarrubea, M., Sorbera, F., \& Crescimanno, G. (2009a). Structure of rat behavior in hole-board: I. Multivariate analysis of response to anxiety. Physiology \& Behavior, 96, 174-179.

Casarrubea, M., Sorbera, F., \& Crescimanno, G. (2009b). Structure of rat behavior in hole-board: II. Multivariate analysis of modifications induced by diazepam. Physiology \& Behavior, 96, 683-692. doi:10.1016/j.physbeh.2009.01.005

Cruz, A. P. M., Frei, F., \& GraefF, F. G. (1994). Ethopharmacological analysis of rat behavior on the elevated plus-maze. Pharmacology, Biochemistry \& Behavior, 49, 171-176.

EsPEJO, E. F. (1997). Structure of the mouse behaviour on the elevated plus-maze test of anxiety. Behavioural Brain Research, 86, 105-112.

EsPEJo, E. F., \& Mir, D. (1993). Structure of the rat's behaviour in the hot plate test. Behavioural Brain Research, 56, 171-176.

EsPejo, E. F., \& Mir, D. (1994). Differential effects of weekly and daily exposure to the hot plate on the rat's behavior. Physiology \& Behavior, $\mathbf{5 5}, 1157-1162$.

EveritT, B. S. (1977). The analysis of contingency tables. New York: Wiley.

Hemerik, L., Bukovinszky, T., Gols, R., van Lenteren, J. C., \& Vet, L. E. M. (2006). Enter the matrix: How to analyze the structure of behavior. Behavior Research Methods, 38, 357-363.

KaluefF, A.V., \& TuohimaA, P. (2004). Grooming analysis algorithm for neurobehavioural stress research. Brain Research Protocols, 13, $151-158$

Kemp, A. S., Fillmore, P. T., Lenjavi, M. R., Lyon, M., ChiczDemet, A., Touchette, P. E., \& Sandman, C. A. (2008). Temporal patterns of self-injurious behavior correlate with stress hormone levels in the developmentally disabled. Psychiatry Research, 157, 181-189.

Kerepesi, A., Jonsson, G. K., Miklósi, A., Topál, J., Csányi, V., \& Magnusson, M. S. (2005). Detection of temporal patterns in doghuman interaction. Behavioural Processes, 70, 69-79.

Kerepesi, A., Kubinyi, E., Jonsson, G. K., Magnusson, M. S., \& MikLósi, A. (2006). Behavioural comparison of human-animal (dog) and human-robot (AIBO) interactions. Behavioural Processes, 73, 92-99.

Lino-De-Oliveira, C., De Lima, T. C. M., \& Carobrez, A. D. P. (2005). Structure of the rat behaviour in the forced swimming test. Behavioural Brain Research, 158, 243-250.

LyON, M., \& KemP, A. S. (2004). Increased temporal patterns in choice responding and altered cognitive processes in schizophrenia and mania. Psychopharmacology, 172, 211-219. 
Magnusson, M. S. (2000). Discovering hidden time patterns in behavior: T-patterns and their detection. Behavior Research Methods, Instruments, \& Computers, 32, 93-110.

Magnusson, M. S. (2004). Repeated patterns in behavior and other biological phenomena. In D. K. Oller \& U. Griebel (Eds.), Evolution of communication systems: A comparative approach (pp. 111-128). Cambridge, MA: MIT Press.

Martaresche, M., Le Fur, C., Magnusson, M., Faure, J. M., \& PICARD, M. (2000). Time structure of behavioral patterns related to feed pecking in chicks. Physiology \& Behavior, 70, 443-451.

Merlet, F., Puterflam, J., Faure, J. M., Hocking, P. M., MagnusSON, M. S., \& PICARD, M. (2005). Detection and comparison of time patterns of behaviours of two broiler breeder genotypes fed ad libitum and two levels of feed restriction. Applied Animal Behaviour Science, 94, 255-271.

Mos, J., Olivier, B., \& van der Poel, A. M. (1987). Modulatory actions of benzodiazepine receptor ligands on agonistic behaviour. Physiology \& Behavior, 41, 265-278.

SHORT, R., \& HORN, J. (1984). Some notes on factor analysis of behavioural data. Behaviour, 90, 203-214.

Spruit, B. M. (1992). Progressive decline in social attention in aging rats: An information-statistical method. Neurobiology of Aging, 13, 145-151.

Spruitt, B. M., \& GisPen, W. H. (1984). Behavioral sequences as an easily quantifiable parameter in experimental studies. Physiology \& Behavior, 32, 707-710.

Takahashi, A., Kato, K., Makino, J., Shiroishi, T., \& Koide, T. (2006). Multivariate analysis of temporal descriptions of open-field behavior in wild-derived mouse strains. Behavior Genetics, 36, 763-774.

Tavaré, S., \& Altham, P. M. E. (1983). Serial dependence of observations leading to contingency tables, and corrections to chi-squared statistics. Biometrika, 70, 139-144.

Van Den Berg, C. L., Van Ree, J. M., \& Spruitt, B. M. (1999). Sequential analysis of juvenile isolation-induced decreased social behavior in the adult rat. Physiology \& Behavior, 67, 483-488.

Vanderschuren, L. J. M. J., Spruitt, B. M., Hol, T., Niesink, R. J. M., \& VAN ReE, J. M. (1996). Sequential analysis of social play behavior in juvenile rats: Effects of morphine. Behavioural Brain Research, 72, 89-95.

van Lier, H., Coenen, A. M. L., \& Drinkenburg, W. H. I. M. (2003). Behavioral transitions modulate hippocampal electroencephalogram correlates of open field behavior in the rat: Support for a sensorimotor function of hippocampal rhythmical synchronous activity. Journal of Neuroscience, 23, 2459-2465.

(Manuscript received October 29, 2008; revision accepted for publication January 14, 2009.) 